CLAWAR 2018: 21st International Conference on Climbing and Walking Robots and the Support Technologies for Mobile Machines, Panama City, Panama, 10-12 September 2018

\title{
ASSISTIVE WHEELCHAIR CASTER UNIT - IMPROVEMENT OF STEP DETECTION METHOD -
}

\author{
S. YOKOTA*, A. MATSUMOTO*, \\ *Department of Mechanical Eng., Toyo University, \\ Kawagoe, Saitama 359-8585, Japan \\ E-mail:s-yokota@toyo.jp, akihiro@toyo.jp \\ D. CHUGO** and H. HASHIMOTO*** \\ **Kwansei Gakuin University, \\ Sanda, Hyogo 669-1337, Japan, E-mail:chugo@kwansei.ac.jp \\ ***Advanced Institute of Industrial Technology \\ Shinagawa-ku, Tokyo 140-0011, Japan, E-mail: hashimoto@aiit.ac.jp
}

\begin{abstract}
The purpose of this research is to develop the assistive caster unit of manual wheelchair for step climbing. This unit is easily replaced with the conventional caster, only a spanner is needed. In addition this caster is simple mechanism, and the power source is only a 006P battery, there is no microcontroller. For realizing this simplicity, The proposed caster unit has two functions to assist the step climbing. One is the assistive plates to enlarge the caster radius imaginarily in the confined front area of the wheelchair, and to enable to climb a higher step. The other is the lock function to fix the caster's swiveling, which realize easy climbing with oblique approach. The lock-function is activated by the detection of the step, and mechanically fixes the caster's swiveling. We have developed some prototypes of this caster unit, so far. However, the problem is still remained in the step detection method being the trigger of activation of the lock function. Therefore, this paper proposes the improved activation method by detecting the step at an instant of touching it.
\end{abstract}

Keywords: mobility assistance; mechanism; wheelchair; step-climbing

\section{Introduction}

The purpose of this research is to develop the assistive caster unit of manual wheelchair for step climbing. This caster unit provides easy step-climbing not only frontal approach but also oblique approach by simple mechanism and a small battery $006 \mathrm{P}$ without any microcontrollers. In addition, this unit is easily replaced with the conventional caster, only a spanner is needed.

There is uneven terrain as one of the mobility barriers for wheelchair 
users, such as steps and stairs. Even a small and slight single step, it becomes a big barrier for mobility of wheelchair users. In order to climb up or passing over a step, the user is required strong arm muscle and technique for wheelie. In addition, in case of oblique step climbing, the more arm muscle is needed. Therefore, the assistive apparatus for step climbing is required.

Based on the above, there are several assistive apparatuses for step climbing $^{1}{ }^{2}$ Auxiliary step-climbing mechanism ${ }^{3}$ being composed by the omnidirectional wheel and auxiliary device were developed. Hinged Caster mechanism ${ }^{4}$ was also developed. These systems showed the good results and improved step climbing performance. However, there are remained problem, in terms of the practical use. The mechanism ${ }^{3}$ uses omnidirectional wheel, and the hinged caster ${ }^{4}$ needs some modifications to the main frame of wheelchair. Moreover, no assistive casters have been developed for oblique step climbing.

On the basis of above, this research develops the assistive caster unit for manual driven wheelchair. This caster unit provides easy step-climbing including oblique approach, namely, driving torque for step climbing can be reduced comparing with conventional caster not only frontal climbing but also oblique climbing. We have developed several prototypes, so far. The first prototype confirmed the effectiveness of above mentioned two functions. ${ }^{5}$ The first prototype used the electromagnetic tooth clutch for configuring lock function. As a result, it needed a large battery and was heavy weight. Thus, the 2 nd prototype improved this problem. ${ }^{6}$ In the 2 nd prototype, a solenoid, rack and pinion gear comprised the lock function. Therefore, it could reduce its weight and the battery voltage and capacity. However, the problem is remained in the step detection method which is the trigger of activation of the lock function. Therefore, this paper proposes the improved activation method by detecting the step at an instant of touching it.

\section{Outline of the assistive caster unit by reviewing 2 nd prototype}

The problems in case of step-climbing are following two points.

Problem 1) The climb-able step height is physically limited by the radius of wheel

Problem 2) In the oblique step-climbing, the driving force is divided, because the caster swivels when the caster faces the step. 
Problem 1) is that: Generally, the climb-able step height is less than $1 / 3$ of a wheel diameter. Thus the large wheel is suited for step-climbing. However, the cater wheel cannot be enlarged, because the attaching portion of it is confined by main frame and footrest of a wheelchair. Thus, diameter of conventional wheelchair is about $100-150 \mathrm{~mm}$. As a result, even a small step (30-40 mm height), users need much power, and have to change the center of weight of his/her body to take a wheelie state.

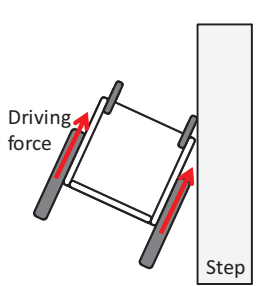

(a)

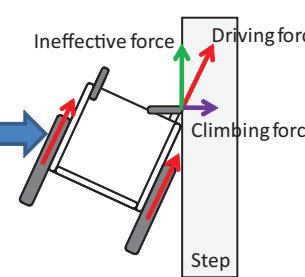

(b)

Fig. 1. Driving force in case of oblique approach to a step

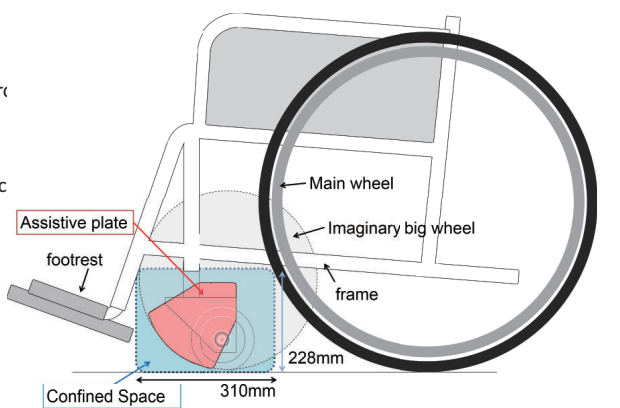

Fig. 2. Assistive plate makes imaginary big caster in the confined space

Problem 2) is that: In case of oblique step climbing (Fig.1(a)), the caster swivels due to the caster dynamics(Fig.1(b)). For this swiveling, driving force being generated by the user is divided into two directions: "moving direction (parallel direction to the step)" and "right direction to the step". The only the force of "right direction" is effective for step climbing. The force "moving direction" is not used, namely the user's force is exhausted. Therefore it is difficult to climb a step obliquely or much power is required(Fig.1(b)).

In order to solve these problems, we have proposed following two functions.

The assistive enlarge the radius of caster wheel as much as possible in the confined space shown in Fig. 2.

Lock-function fix the caster's swiveling in case of oblique step climbing.

Figure $3^{6}$ shows the 2nd prototype having these two functions. The black plates in both sides are the assistive plates. This plate has an equivalent climbing ability to big wheel in confined attachment space. Figure 6 shows 


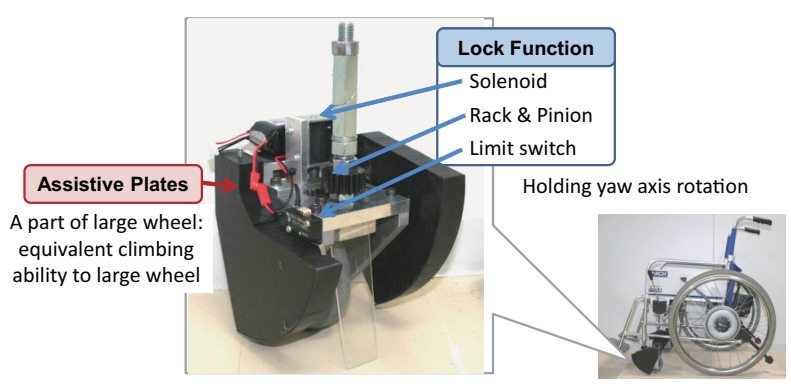

Fig. 3. Assistive Wheelchair Caster Unit (2nd prototype)

the movement of assistive plates. After the plates touch the step, they rotate, and the caster unit climb the step.

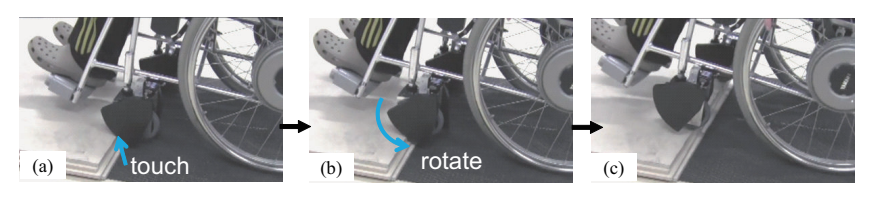

Fig. 4. Movement of the assistive plates

The lock function is the portion consisting of a solenoid, a limit switch and rack \& pinion gears. It holds the caster's swiveling, and assists the oblique step climbing. Figure 5 shows the movement of the lock function. First, (1)After the assistive plates touch the step, they rotates, and step climbing begins. (2) The limit switch detects the rotation of the assistive plates, and (3) current flows into solenoid. (4)The solenoid is activated, and pulls up the rack gear, and then (5) the pinion gear and rack gear are engaged. (6) Finally, the caster's swiveling is fixed.

The performance of this prototype was tested by the experiment. ${ }^{7}$ In the experiment, the load cell was sandwiched between main wheel and the hand rim, and the strain was used as the index of user's driving force.

First, the efficiency of assistive plates was tested. The strain at the load cell was measured at the step height of 20,30, $40 \mathrm{~mm}$ in both cases: with assistive plates and without assistive plates. The results are summarized in Table 1. In the table, "X" indicates that the step climbing was failed. The table shows that the asssitive plates could reduce the driving force in all step height. 


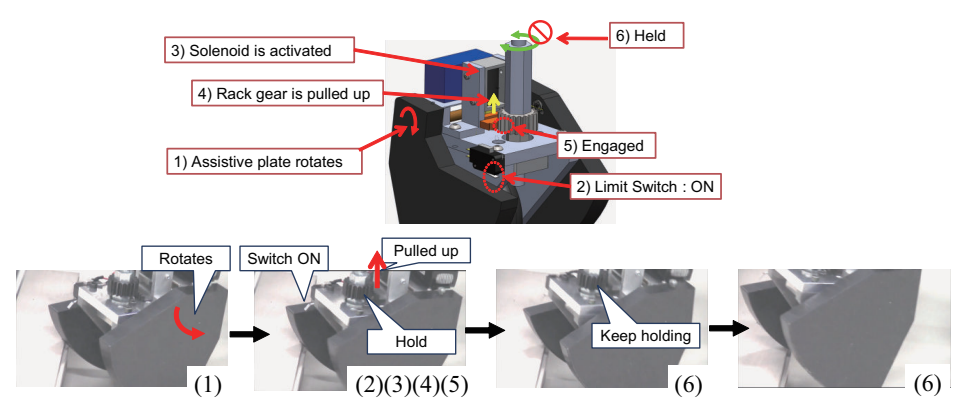

Fig. 5. Movement of the Lock function

Table 1. Maximum strain $\left[10^{-6}\right]$ Table 2. Maximum strain[10 $\left[10^{-6}\right]$ with aswith/without assistive plates (approach an- sitive plates, oblieque step climing at $\pi / 4$ gle $=0[\mathrm{rad}])$

\begin{tabular}{|l||l|l|}
\hline $\begin{array}{l}\text { Step } \\
\text { height } \\
{[\mathrm{mm}]}\end{array}$ & $\begin{array}{l}\text { Without } \\
\text { Assistive } \\
\text { plates }\end{array}$ & $\begin{array}{l}\text { With As- } \\
\text { sistive } \\
\text { plates }\end{array}$ \\
\hline \hline 20 & 73.91 & 54.13 \\
\hline 30 & 102.38 & 58.32 \\
\hline 40 & $\mathrm{X}$ & 59.88 \\
\hline
\end{tabular}
$[\mathrm{rad}]$

Next, the lock function was tested. The strain at the load cell was also measured in the cases: with lock function and without lock function. The result is shown in Table 2. The table shows that the lock function could reduce the users' driving force at the oblique step climbing.

Therefore, it is confirmed that the two functions of our prototype could assist step climbing by the simple mechanism.

\section{Problem of 2nd Prototype}

Basically the proposed two functions are effective for step climbing. However, in 2nd prototype, occasionally the lock function did not activate properly, and the caster's swiveling occurred when oblique climbing. The cause of this is that the prototype structurally can not instantaneously activate the lock function at the moment the caster unit touches the step. The rotation of the assistive plates were used to detect the step. The limit switch detects this rotation, and activates lock function. Rotating the assistive plates means that step climbing has already been started. Therefore, the problem has been remained that the caster can not be locked at the start 
of climbing where the greatest user's force is required.
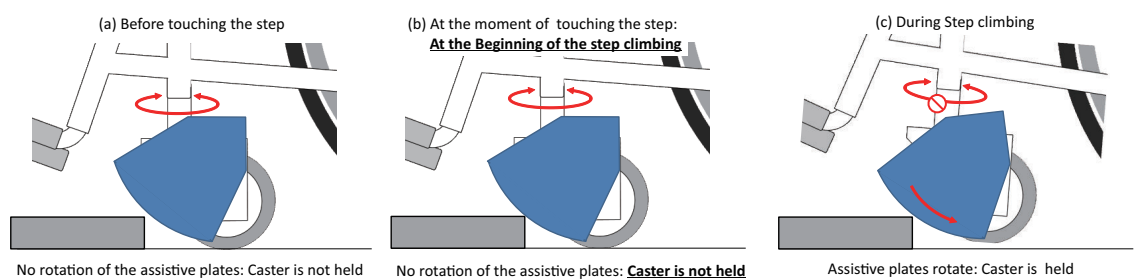

Fig. 6. Problem of 2nd prototype: Step detection

\section{Improvement}

In order to solve the above mentioned problem, it is required not to detect the step by rotation of the assistive plate, but to detect the step at the moment the assistive plate touches the step. Therefore, we consider a mechanism for detecting whether or not the arc portion of the assistive plate touches the step. This mechanism realizes instantaneous activation of the lock function at the moment the caster unit touches the step. This detection needs to detect only the contact between the assistive plates and the step, and it is not necessary to detect the contact position on the arc portion of the assistive plates. In order to meet this requirement and easily detect the step, We consider the tape switch (141 - BPH, Tape Switch Japan Co., Ltd.) to be placed on the arc of the assistive plate (Fig. 7).

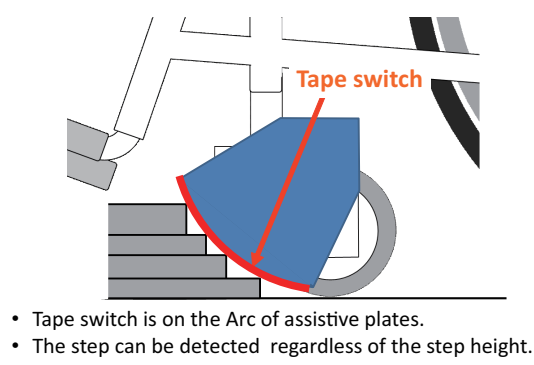

Fig. 7. Attaching portion of the tape swiche

The 3rd prototypewas made shown in Fig. 8). Parts of the 2nd prototype 
were diverted and the tape switch was affixed to the circular arc portion of the assistive plates.

Experiments were conducted to confirm the superiority of step climbing by this new step detection method. In the experiments, the forces required to oblique step climbing were measured by using a force gauge in both cases of $2 \mathrm{nd}$ and $3 \mathrm{rd}$ prototype. The height of the step was set to $40 \mathrm{~mm}$, and the oblique approach angle was set to $\theta=\pi / 3$ [rad]. Under these conditions, the measurements were made ten times using 2 nd and 3re prototype, respectively.

The result is shown in Fig. 9. From this result, it can be seen that the proposed new prototype (3re Ver.) has a smaller driving force. Therefore, by locking the caster's swiveling simultaneously with the contact of the step, it can be said that the caster's swiveling was locked before the step climbing and the driving force of user could be reduced.

From the above, by improving the step detection method, the delay of the lock function was gotten rid of, the driving force could be reduced.

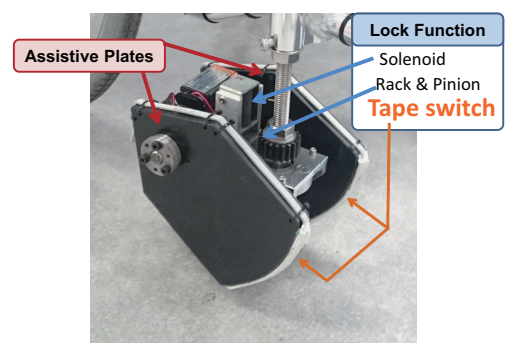

Fig. 8. Overview of the 3rd prototype

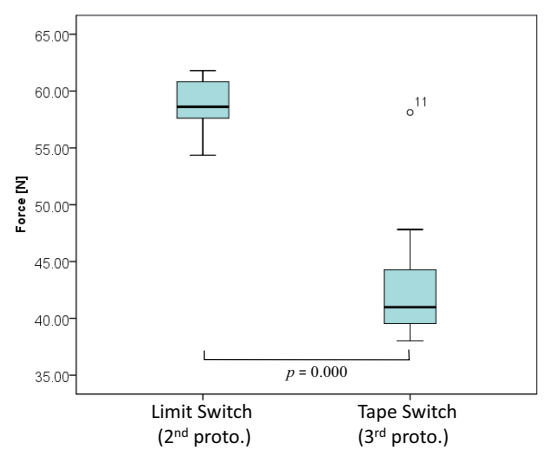

Fig. 9. Result of the force of step climbing

\section{Conclusion}

This research develops the assistive caster unit for manual driven wheelchair. This caster unit provides easy step-climbing including oblique approach.This caster unit has two key functions. One is the assistive plates to enlarge the caster radius imaginarily in the confined front area of the wheelchair, and to enable to climb a higher step. The other is the lockfunction to fix the swiveling, which realize easy climbing with oblique ap- 
proach. The lock-function is activated by the detection of the step, and mechanically fixes the swiveling of the caster. We have developed some prototypes of this caster unit, so far. However, the problem was still remained in the step detection method being the trigger of activation of the lockfunction. Therefore, this paper proposed the improved activation method of lock function by detecting the step at the moment of touching it.

The improved method was to attach the tape switch on the circular arc portion of assistive plate as a new step detection method. The experimental result showed that the caster's swiveling was locked before the step climbing and the driving force of user could be reduced by locking the caster's swiveling simultaneously with the contact of the step. Therefore, the delay of the lock function was gotten rid of, the driving force could be reduced by improving the step detection method.

\section{References}

1. Y. Sugahara, N. Yonezawa and K. Kosuge, A novel stair-climbing wheelchair with transformable wheeled four-bar linkages, in Proc. of the 2010 IEEE/RSJ International Conference on Intelligent Robots and Systems (IROS 2010), (Taipei, Taiwan, 2010).

2. S. Yu, T. Wang, X. Li, C. Yao, Z. Wang and D. Zhi, Configuration and tip-over stability analysis for stair-climbing of a new-style wheelchair robot, in Proc. of the 2010 IEEE International Conference on Mechatronics and Automation (ICMA 2010), (Xi'an, China, 2010).

3. T. Hayashi, S. Kawakami, H. Matsuo and M. Yuinamochi, A study on a multifunction wheelchair with auxiliary step-climbing mechanism, in JSME Proceedings of the Welfare Engineering Symposium 2003, (Tokyo, Japan, 2003).

4. T. Ohmura, T. Iwamoto and K. Shibuya, Step traveling of wheel chair with hinged caster mount, in Proc. of the 2006 JSME conference on Robotics and Mechatronics, (Tokyo, Japan, 2006).

5. S. Yokota, T. Ito, T. Yamaguchi, D. Chugo and H. Hashimoto, An assistive wheelchair caster unit for step climbing, in Proc. of the 37th Annual Conference of the IEEE Industrial Electronics Society (IECON 2011), (Beijing, China, 2011).

6. S. Yokota, H. Hashimoto, D. Chugo and K. Kawabata, Improvement of assistive wheelchair caster unit for step climbing, in Proc. of the 21st IEEE International Symposium on Robot and Human Interactive Communication Ro-Man 2012, (Paris, France, 2012).

7. S. Yokota, D. Chugo, H. Hashimoto and K. Kawabata, Journal of Mechanical Engineering and Automation 3, 38 (2013). 